\title{
True-Value Regression with Non-Response
}

\author{
Gordon G. Bechtel \\ University of Florida and Florida Research Institute
}

\begin{abstract}
True-value theory (Bechtel, 2010), as an extension of randomization theory, allows arbitrary measurement errors to pervade a survey score as well as its predictor scores. This implies that true scores need not be expectations of observed scores and that expected errors need not be zero within a respondent. Rather, weaker assumptions about measurement errors over respondents enable the regression of true scores on true predictor scores. The present paper incorporates Särndal-Lundström (2005) weight calibration into true-value regression. This correction for non-response is illustrated with data from the fourth round of the European Social Survey (ESS). The results show that a true-value regression coefficient can be corrected even with a severely unrepresentative sample. They also demonstrate that this regression slope is attenuated more by measurement error than by non-response. Substantively, this ESS analysis establishes economic anxiety as an important predictor of life quality in the financially stressful year of 2008.
\end{abstract}

Key words: Census totals, cross-national micro data, distribution-free regression, economic anxiety, equation error, errors in variables, life quality, respondent-specific weight calibration, true and observed scores.

\section{Introduction}

The collection of micro data, particularly with opinion polls, has become a world-wide commercial and governmental activity. It is well known, however, that measurement error distorts opinion regressions by attenuating their coefficients. The error score on an opinion scale is the average of the errors on the items making up that scale. Bechtel (2010) showed that this error score, in contrast to that in psychological test theory (Lord and Novick, 1968, Chapter 2), need not have expectation zero within a particular survey respondent. A broader psychogenesis of a single person's error score recognizes that it contains her (his) fixed item biases as well as random item errors. Moreover, classic assumptions about the behavior of these error scores over survey respondents (Gulliksen, 1950, pp. 4-7), 
enable the regression of true opinion scores on their true predictor scores. This true-value regression corrects predictor slopes for attenuation due to score error.

A second unwanted phenomenon biasing micro-survey regressions is unit nonresponse. This distorts regression coefficients by reducing a representative sample of respondents to an unrepresentative sub-sample. The present paper extends the reach of true-value theory by treating unit non-response with Särndal-Lundström (2005) calibration of sampling weights. SL calibration is respondent-specific and, therefore, more precise than subgroup-specific weighting-class and poststratification adjustments of sampling weights (Lohr, 1999, pp. 266-271). It enables true-value theory to tackle unit non-response as well as the other untoward effects of item non-response and measurement error. The corrective value of SL calibration is demonstrated with data from the fourth round of the European Social Survey (ESS). Substantively, this exercise confirms economic anxiety as an important predictor of life quality in the global financial crisis of 2008 .

Section 2 describes true and observed scores for life quality and economic anxiety. Section 3 distinguishes a population of true scores from a realized census of observed scores. Our target coefficients are defined by an OLS regression over the population of true scores. Our realized census links its sample to these target coefficients. Section 4 describes two successive adjustments for unit non-response in a sample from our realized census, i.e. a weighting-class adjustment and a respondent-specific SL calibration. The resulting estimates of census totals, which are weighted sample totals, make up the regression coefficients given in Section 5 .

Sections 6 and 7 apply calibrated true-value regression to data from the fourth round of the European Social Survey (ESS). The effect of economic anxiety on life quality is estimated from a criterion sample and a non-representative sub-sample. This exercise demonstrates the corrective power of SL weight calibration in the presence of unit non-response. It also shows that the effect of unit non-response on a regression slope is not as strong as that of measurement error in our predictor score. Section 8 stresses the advantages of true value theory in analyzing opinion surveys with measurement error and non-response.

\section{True and Observed Scores}

Using the ESS 2004, T. Bechtel (2007) showed that psychological and economic distress are important predictors of English and German life quality. This finding begs the question about pan-European effects of economic anxiety on life quality in the subsequent global financial crisis. The present study regresses a life-quality score on an economic-anxiety score derived from items in the fourth round of the European Social Survey (ESS) administered in the financially stressful year of 2008. These items are exhibited in Table 1. All item responses are 
coded to range between 0 and 10. An individual's life-quality score is the same single-item rating of life satisfaction used by T. Bechtel (2007). However, his single-item economic anxiety scale, i.e. the last item in Table 1, is extended here to include the second and third items in the table, which were not available in the 2004 ESS. Thus, our economic anxiety score is the average of three separate item ratings. This 3-item score also takes values between 0 and 10 .

Table 1: Single and multiple-item scales

\section{Life quality}

All things considered, how satisfied are you with your life as a whole nowadays?

Extremely dissatisfied 012345678910 Extremely satisfied

\section{Economic anxiety}

During the next 12 months how likely is it that there will be some periods when you don't have enough money to cover your household necessities?

Not at all likely $0 \quad$ Not very likely $3.33 \quad$ likely $6.67 \quad$ Very likely 10

During the next 12 months how likely is it that you will not receive the health care you really need if you become ill?

Not at all likely $0 \quad$ Not very likely $3.33 \quad$ likely $6.67 \quad$ Very likely 10

How do you feel about your household's income nowadays?

$\begin{array}{llll}\text { Living comfortably } 0 & \text { Coping } 3.33 \quad \text { Difficult } 6.67 & \text { Very difficult } 10\end{array}$

Source: The items in this table are found on the website ess.nsd.uib.no

The life-satisfaction rating of respondent $i$ in country $k$ is modeled as

$$
Y_{k i}=\eta_{k i}+E_{k i}
$$

where $\eta_{k i}$ is $k i$ 's true item score in the interval $[0,10]$. Respondent $k i$ 's measurement error $E_{k i}$ on the life-quality item in Table 1 may be a fixed bias, a random bias, or a random error with expectation zero. This error score may have nonzero expectation, and $k i$ 's expected observed score on this survey item need not be her (his) true item score $\eta_{k i}$.

Referring to the economic-anxiety scale in Table 1 , respondent $k i$ 's three ratings are modeled as

$$
\begin{array}{ll}
X_{k i 1}=\tau_{k i}+U_{k i 1}, & \text { (money for household necessities) } \\
X_{k i 2}=\tau_{k i}+U_{k i 2}, & \text { (receive health care needed) } \\
X_{k i 3}=\tau_{k i}+U_{k i 3} . & \text { (household's income nowadays) }
\end{array}
$$

Here $\tau_{k i}$ is $k i$ 's true economic anxiety in the interval [0,10], and $U_{k i 1}, U_{k i 2}$, and $U_{k i 3}$ are her (his) item measurement errors. Again, each item error may be a 
fixed or random bias or a random error with expectation zero. Individual ki's observed economic-anxiety score is

$$
\begin{aligned}
X_{k i} & =\left(X_{k i 1}+X_{k i 2}+X_{k i 3}\right) / 3 \\
& =\tau_{k i}+\left(U_{k i 1}+U_{k i 2}+U_{k i 3}\right) / 3 \\
& =\tau_{k i}+U_{k i},
\end{aligned}
$$

where $U_{k i}$ is her (his) error score. Allowing item measurement errors to have nonzero expectation implies that the error score $U_{k i}$ may also have nonzero expectation. Thus, once again, the expectation of $k i$ 's observed score $X_{k i}$ in (2.2) need not be her (his) true score $\tau_{k i}$. This weakens the assumptions of psychological test theory (Lord and Novick, 1968, Chapter 2), permitting a more realistic psychogenesis of a survey respondent's error score.

\section{True-Value Population Theory}

\subsection{Cross-National Population Regression}

Referring to equations (2.1) and (2.2), we make inferences to the population $\left\{\eta_{k i} \tau_{k i} \mid k=1, \cdots, 16 ; i=1, \cdots, N_{k}\right\}$ of true scores. Our cross-national population model is

$$
\eta_{k i}=\beta_{0}+\beta_{1} \tau_{k i}+\varepsilon_{k i}, \quad \text { for } k=1, \cdots, 16 \text { and } i=1, \cdots, N_{k},
$$

where $\varepsilon_{k i}$ is equation error in a simple OLS regression of the population of true scores $\eta_{k i}$ on the population of true scores $\tau_{k i}$. The coefficient vector $\boldsymbol{\beta}=\left(\beta_{0} \beta_{1}\right)^{\top}$ is identified as

$$
\boldsymbol{\beta}=\left(\sum_{p} \mathbf{T}_{k i} \mathbf{T}_{k i}^{\boldsymbol{\top}}\right)^{-1} \sum_{p} \mathbf{T}_{k i} \eta_{k i}
$$

where the two population summations run over $i=1, \cdots, N_{k}(k=1, \cdots, 16)$, and the row vector $\mathbf{T}_{k i}^{\top}=\left(1 \tau_{k i}\right)$.

\subsection{An Estimable Expression of $\beta$}

We distinguish the cross-national population $\left\{\eta_{k i} \tau_{k i} \mid k=1, \cdots, 16 ; i=\right.$ $\left.1, \cdots, N_{k}\right\}$ of true scores from a cross-national census $\left\{Y_{k i} X_{k i} \mid k=1, \cdots, 16\right.$; $\left.i=1, \cdots, N_{k}\right\}$ of realized scores. Departures of realized scores from true scores, as seen in equations (2.1) and (2.2), are due to measurement error.

Because the true scores $\eta_{k i}$ and $\tau_{k i}$ are unobservable, an estimable form of $\beta$ is obtained by assuming that over the census error scores sum to zero, are uncorrelated with true scores, and are uncorrelated with each other. Under these 
classic assumptions (Gulliksen, 1950, pp. 4-7), along with (2.1) and (2.2), Bechtel (2010) shows that the target parameter (3.2) may be written as

$$
\boldsymbol{\beta}=\left(\sum_{p} \mathbf{X}_{k i} \mathbf{X}_{k i}^{\top}-\Delta\right)^{-1} \sum_{p} \mathbf{X}_{k i} Y_{k i},
$$

where $\mathbf{X}_{k i}^{\top}=\left(1 X_{k i}\right), \Delta=\operatorname{diag}(0, \delta)$, and

$$
\delta=\sum_{p} U_{k i}^{2}=(1-\alpha)\left\{\sum_{p} X_{k i}^{2}-\left(\sum_{p} X_{k i}\right)^{2} / N\right\} .
$$

The first diagonal element of $\Delta$ in (3.3) is zero because the intercept in (3.1) multiplies an error-free unit value. The $\alpha$ in (3.4) is the census coefficient alpha for our economic anxiety score. (See Bechtel, 2010, formula 3.3) (See also Cronbach, 1951; Lord and Novick, 1968, Chapter 4; Nunnally and Bernstein, 1994; and StataCorp., 2001)

Estimates of the census totals in (3.3) and (3.4) are now developed in Sections 4 and 5 with a sample from this cross-national census.

\section{Weighting a Cross-National Sample}

\subsection{A Weighting-Class Adjustment for Unit Non-Response}

Let a sample of size $n_{k}$ be drawn without replacement from the census in country $k$ such that $\pi_{k i}$ is the probability of individual $i$ 's sample inclusion ( $k=$ $\left.1, \cdots, 16 ; i=1, \cdots, N_{k}\right)$. Let $m_{k}$ be the number of responding individuals in the sample selected for country $k$. For each respondent $i$ in country $k$ the ESS provides a design weight $m_{k} / \pi_{k i} \sum_{r} \pi_{k i}^{-1}$, where the summation over responding individuals runs over $i=1, \cdots, m_{k}\left(<n_{k}\right)$. These normed inverses of the sample inclusion probabilities sum to $m_{k}$ for each country $k$.

For pan-European work the ESS multiplies its design weights by each country's population size weight to construct weights representative of the crossnational population being studied. Country $k$ 's population size weight is $\sum_{s} \pi_{k i}^{-1}$ / $10000 m_{k}$, where $\sum_{s} \pi_{k i}^{-1}=N_{k}$ is its population size aged 15 and over. Thus, the pan-European weight for individual $i$ in country $k$ is

$$
\begin{aligned}
w_{k i} & =\left(m_{k} / \pi_{k i} \sum_{r} \pi_{k i}^{-1}\right)\left(\sum_{s} \pi_{k i}^{-1} / 10000 m_{k}\right) \\
& =\left\{10000 \pi_{k i}\left(\sum_{r} \pi_{k i}^{-1} / \sum_{s} \pi_{k i}^{-1}\right)\right\}^{-1},
\end{aligned}
$$

where the summation over selected individuals in country $k$ runs over $i=1, \cdots$, $n_{k}$, and the summation over responding individuals runs over $i=1, \cdots, m_{k}$. The 
country-specific ratio $\sum_{r} \pi_{k i}^{-1} / \sum_{s} \pi_{k i}^{-1}$ in (4.1) is an estimate of the probability of a selected individual in country $k$ participating in its survey. The product of this ratio and $\pi_{k i}$ estimates the probability of individual $i$ in country $k$ being selected and participating in the 2008 ESS.

In the special case of complete sample response, the ratio in (4.1) is $\sum_{s} \pi_{k i}^{-1} /$ $\sum_{s} \pi_{k i}^{-1}(=1)$, and $w_{k i}$ is the normed sampling weight $1 / 10000 \pi_{k i}$. In the presence of non-response the multiplier $\sum_{s} \pi_{k i}^{-1} / \sum_{r} \pi_{k i}^{-1}(>1)$ increases $1 / 10000 \pi_{k i}$. Hence, with non-response country $k$ acts as a weighting class, and $\sum_{s} \pi_{k i}^{-1} / \sum_{r} \pi_{k i}^{-1}$ is a weighting-class adjustment of the normed sampling weight $1 / 10000 \pi_{k i}$ (Lohr, 1999, pp. 266-268).

\subsection{Särndal-Lundström (SL) Calibration of the $w_{k i}$}

The pan-European weight in (4.1) insures that the respondents in a crossnational sample represent countries in proportion to their population sizes. However, like all weighting-class adjustments, it assumes the same probability of response for every individual $i$ in country $k$. Therefore, we turn to a calibration of (4.1) which provides a respondent-specific adjustment to $w_{k i}$.

Let $\mathbf{Z}_{k i}^{\top}=\left(Z_{k i 1} Z_{k i 2}\right)$ be a row vector of two auxiliary variables for $k=$ $1, \cdots, 16$ and $i=1, \cdots, n_{k}$. In the present study $Z_{k i 1}$ and $Z_{k i 2}$ are unhappiness and lowness of income. These variables are associated with unit non-response and our study variables economic anxiety and life quality. Following Särndal and Lundström (2005, Chapter 6) we seek weights $c_{k i}$ that satisfy the calibration equation

$$
\sum_{r} c_{k i} \mathbf{Z}_{k i}=\sum_{s} w_{k i} \mathbf{Z}_{k i} .
$$

The sum on the right of $(4.2)$, which is over $i=1, \cdots, n_{k}(k=1, \cdots, 16)$, estimates the census total $\sum_{p} \mathbf{Z}_{k i}$ up to a scale factor. Therefore, equation (4.2) calibrates the weights $w_{k i}$ to new weights $c_{k i}$ such that $\sum_{r} c_{k i} \mathbf{Z}_{k i}$, which is over $i=1, \cdots, m_{k}\left(<n_{k}\right)(k=1, \cdots, 16)$, also estimates the census total of auxiliary vectors. This upward scaling of $w_{k i}$ in (4.1) to the calibrated weight $c_{k i}$ is given by

$$
c_{k i}=\left(1+\boldsymbol{\lambda}^{\top} \mathbf{Z}_{k i}\right) w_{k i}
$$

where the multiplier in parentheses is linearly dependent on individual $k i$ 's auxiliary vector $\mathbf{Z}_{k i}$. This multiplier is found by substituting $c_{k i}$ in (4.3) into (4.2) and solving for $\boldsymbol{\lambda}^{\top}$. Thus

$$
\boldsymbol{\lambda}^{\top}=\left(\sum_{s} w_{k i} \mathbf{Z}_{k i}-\sum_{r} w_{k i} \mathbf{Z}_{k i}\right)^{\top}\left(\sum_{r} w_{k i} \mathbf{Z}_{k i} \mathbf{Z}_{k i}^{\top}\right)^{-1}
$$

which is computed from the pan-European weights and the auxiliary data (cf. Särndal and Lundström, 2005, pp. 57-59). 


\section{Estimating $\beta$ as a Function of SL Estimators}

The SL estimators of the census totals $\sum_{p} \mathbf{X}_{k i} \mathbf{X}_{k i}^{\top}$ and $\sum_{p} \mathbf{X}_{k i} Y_{k i}$ in (3.3) are given (up to multiplication) by $\sum_{r} c_{k i} \mathbf{X}_{k i} \mathbf{X}_{k i}^{\top}$ and $\sum_{r} c_{k i} \mathbf{X}_{k i} Y_{k i}$. Thus, in the presence of non-response and measurement error, the estimator of our target parameter $\boldsymbol{\beta}$ in (3.2) and (3.3) is

$$
\mathbf{B}=\left(\sum_{r} c_{k i} \mathbf{X}_{k i} \mathbf{X}_{k i}^{\top}-D\right)^{-1} \sum_{r} c_{k i} \mathbf{X}_{k i} Y_{k i} .
$$

In (5.1) the two summations over respondents run over $i=1, \cdots, m_{k}(k=$ $1, \cdots, 16)$, and the matrix $D=\operatorname{diag}(0, d)$, where

$$
d=(1-a)\left\{\sum_{r} c_{k i} \mathbf{X}_{k i}^{2}-\left(\sum_{r} c_{k i} \mathbf{X}_{k i}\right)^{2} / \sum_{r} c_{k i}\right\} .
$$

In (5.2) the three weighted totals over respondents are SL estimators of their respective census totals in (3.4). The coefficient $a$ is an estimate of the census $\alpha$ in (3.4). This estimate is given by Bechtel (2010, formula 4.1) using the SL calibrated weights in (4.3). Table 2 exhibits this estimated alpha coefficient for our ESS criterion sample and an unrepresentative sub-sample in 2008.

Formula (5.1) points up two major corrections that true-value theory adds to design-based regression. First, the diagonal matrix under the inverse in (5.1) corrects the well-known design-based formula, which holds in the absence of measurement error when $D=0$ (Nathan, 1988, p. 255; Lohr, 1999, p. 360). Second, the calibrated weights $c_{k i}$ in $(5.1)$ correct $\mathbf{B}$ for unit non-response. These calibrated weights replace the sampling weights in the classical design-based formula.

Finally, the standard errors of the coefficients $B_{0} B_{1}$ of $\mathbf{B}$ in (5.1) are computed by an iterative procedure derived by Bechtel (2010, Appendix). The corrected standard errors for our unrepresentative ESS sub-sample are exhibited in the right-hand column of Table 3.

\section{Study Design}

\subsection{Data}

The data for our analysis was supplied by the European Social Survey (Jowell and the Central Co-ordinating Team, 2008). This is

... an academically-driven social survey designed to chart and explain the interaction between Europe's changing institutions and the attitudes, beliefs and behaviour patterns of its diverse populations. Now moving into its fourth round, the survey covers over 30 nations and employs the most rigorous methodologies. The survey has been funded through the European 
Commission's fifth and sixth Framework Programme, the European Science Foundation and national funding bodies in each country $\cdots$.

Data collection takes place every two years, by means of face to face interviews of around an hour in duration $\cdots$. The questionnaire consists of a 'core' module lasting about half an hour which remains relatively constant from round to round $\cdots$ the core module aims $\cdots$ to monitor change and continuity in a wide range of socio-economic, socio-political, socio-psychological and socio-demographic variables.

(www.europeansocialsurvey.org)

The present study uses items from the ESS that were administered during the global financial crisis of 2008. We carry out a pan-European analysis that includes 16 countries; namely, Belgium, Switzerland, Germany, Denmark, Spain, Finland, France, Great Britain, Norway, Portugal, Sweden, Poland, Hungary, Slovenia, Bulgaria, and Russia. In each country a representative probability sample was drawn from the residential population aged 15 and older. (www.europeansocialsurvey.org)

\subsection{Imputation for Item Non-Response}

Missing item responses in the ESS dataset were filled in with imputed values. If a respondent's score includes item imputations, it will differ from her (his) census score. These score differences will (approximately) sum to zero over a large sample. Thus, in the presence of imputation the calibrated totals in Sections 4.2 and 5 will differ negligibly from those that would be obtained with census imputations instead of sample imputation (Bechtel, 2010).

Referring to Table 1, one regression imputation was carried out for the single-item scale measuring life satisfaction. This scale was regressed on unhappiness, gender, age, and education. For the three-item economic anxiety scale, three regression imputations were conducted using those items making up this scale. Thus, each item was regressed on the two other items, as well as on gender, age, and education, to impute its missing responses (cf. StataCorp., 2001, Volume 2, pp 69-71).

Referring to equation (2.1), the single regression imputation for life satisfaction filled in missing $Y_{k i}$ scores. For equation (2.2) the three regression imputations for economic anxiety filled in missing $X_{k i 1}, X_{k i 2}$ and $X_{k i 3}$ scores. Subsequently, the derived $X_{k i}$ score was computed as the average of these three observed (and imputed) item scores. These regression imputations avoided sample loss by preserving all of the 16-nation dataset in the 2008 ESS.

\subsection{Criterion and Comparative Regressions}

The present study assesses the SL correction for non-response over and above the separate correction for measurement error in true-value regression (Bechtel, 2010). To make this evaluation we carry out a true-value regression on an unrepresentative ESS sub-sample using the $c_{k i}$ in (4.3). The resulting effect of economic anxiety on life quality is then compared with (a) that obtained from the same the unrepresentative sub-sample using the $w_{k i}$ in (4.1) and (b) that obtained from a criterion sample. 
Our criterion sample consists of those respondents who reported income in the 2008 ESS. From this sample we artificially draw a sub-sample that is severely biased with respect to income and emotional distress. This sub-sample is constructed by deleting $60 \%$ of low income respondents and $60 \%$ of unhappy respondents. The auxiliary variables $Z_{k i 1}$ and $Z_{k i 2}$ (in Section 4.2) are the reversed income and happiness scales in the ESS. These scales meet Särndal and Lundström's (2005, p. 110) guidelines for the selection of auxiliary variables; namely, $Z_{k i 1}$ and $Z_{k i 2}$ explain non-response, and they also explain life quality and economic anxiety.

\section{Results}

\subsection{Reliability of the Economic Anxiety Score}

Estimates of the census coefficient alpha, computed from formula 4.1 in Bechtel (2010), are exhibited in Table 2. The pan-European weights $w_{k i}$ in (4.1) here are used for the alpha estimate in the criterion sample. In the unrepresentative sub-sample this estimate is computed with the pan-European weights $w_{k i}$ in (4.1) and with the SL weights $c_{k i}$ in (4.3). Table 2 shows that the estimate of the census alpha is not affected by biased sampling or type of weighting.

Table 2: Alpha coefficients for the economic anxiety score

\begin{tabular}{lcc}
\hline & $\begin{array}{c}\text { Criterion } \\
\text { sample }\end{array}$ & $\begin{array}{c}\text { Unrepresentative } \\
\text { sub-sample }\end{array}$ \\
\hline With weights $w_{k i}$ in $(4.1)$ & .778 & .765 \\
With weights $c_{k i}$ in $(4.3)$ & - & .777 \\
\hline
\end{tabular}

\subsection{The Effect of Economic Anxiety on Life Quality}

Unit nonresponse. The first row of Table 3 shows the true-value regression effects for our criterion sample with the $w_{k i}$, our biased sub-sample with the $w_{k i}$, and our same biased sub-sample with the $c_{k i}$. These cross-national regression coefficients were computed from formula (5.1) with the weights indicated in the third row of Table 3 . The imputed sample sizes for these regressions appear in the last row of the table.

The second row in Table 3 exhibits the classic design-based regression estimates (Lohr, 1999, p. 360), which are a special case of (5.1) when $D=0$. These coefficients are also computed for our criterion and biased samples with the weights indicated in the third row of Table 3 .

The estimates in the first and second rows of Table 3 show that both the true-value and design-based slopes are attenuated in the unrepresentative sub-sample using the pan-European weights $w_{k i}$ in (4.1). This attenuation, however, is repaired by the SL calibrated weights $c_{k i}$ in (4.3) which remove most of the non-response bias.

Measurement error in the economic anxiety score. Each of the three columns in Table 3 shows an (absolute) difference of .12 between the true-value regression slope and the design-based regression slope. Thus, the effect of economic anxiety on life quality is 
sharply attenuated by measurement error in the economic anxiety scores. This attenuation is corrected by formula (5.1) using the $w_{k i}$ in (4.1) and the $c_{k i}$ in (4.3). Finally, the standardization of $Y_{k i}$ in (2.1) and $X_{k i}$ in (2.2) allows the interpretation of each slope in Table 3 as a loss in life quality on the scale $[0,10]$ due to a one-unit gain in economic anxiety on this same scale. The results in Table 3 show that estimates of this loss are more severely attenuated by measurement error than by unit non-response.

Table 3: Effects of economic anxiety on life quality

\begin{tabular}{lccc}
\hline & $\begin{array}{c}\text { Criterion } \\
\text { sample }\end{array}$ & $\begin{array}{c}\text { Unrepresentative } \\
\text { sub-sample }\end{array}$ & $\begin{array}{c}\text { Unrepresentative } \\
\text { sub-sample }\end{array}$ \\
\hline True-value regression & $-.565(.015)^{1}$ & $-.493(.026)$ & $-.539(.027)$ \\
Design-based regression & $-.439(.011)$ & $-.377(.018)$ & $-.419(.020)$ \\
\hline Weight used & $w_{k i}$ in $(4.1)$ & $w_{k i}$ in $(4.1)$ & $c_{k i}$ in $(4.3)^{2}$ \\
Imputed sample size & 24197 & 13029 & 13029 \\
\hline
\end{tabular}

\footnotetext{
${ }^{1}$ All regression coefficients are significant beyond the .000 level. Their standard errors are in parentheses.

${ }^{2}$ The calibrated weights $c_{k i}$ in (4.3) are computed using the auxiliary variables lowness of income and unhappiness.
}

\section{Advantages of True-Value Regression in Opinion polling}

\subsection{Weakening Psychological Test Theory}

True-value theory weakens psychological test theory (Lord and Novick, 1968, Chapter 2) by allowing a single respondent's error on a survey item to have nonzero expectation. In addition to the usual random error with zero expectation, a measurement error can be a fixed or random item response bias. This acknowledges a broader psychogenesis of item error within a survey respondent.

\subsection{Weakening Randomization Theory}

True-value theory also weakens the assumption in design-based sampling that a finite population is a set of errorless constants (Nathan, 1988; Lehmann, 1999; Lohr, 1999; Chaudhuri and Stenger, 2005). This conventional population, which is untenable for opinion polling, is replaced by two finite sets of vectors. The first set is a population of true vectors, and the second is a census of realized vectors. The interpretation of the realized scores $Y_{k i}$ and $X_{k i}$ in (2.1) and (2.2) as deviations from the true scores $\eta_{k i}$ and $\tau_{k i}$, rather than errorless constants, is a realistic relaxation of randomization theory (Neyman, 1934; Bellhouse, 1988; Thompson, 1997). Classic assumptions about these deviations (error scores) across the census, stated in Section 3.2 and Gulliksen (1950, pp. 4-7), enable us to regress a true opinion score on a true predictor score.

\subsection{Distribution-Free Regression for Micro-Data}


By stopping short of assumptions about the distributions of $\tau_{k i}$ and $U_{k i}$ in $(2.2)$ across the census, we establish a middle ground between design- and model-based regression. This avoids formulating our census as a sample from a "super-population" with a specified distribution (Binder, 1983; Skinner, Holt and Smith, 1989; Valliant, Dorfman and Royall, 1999). To date the treatment of measurement error in linear regression has been dominated by model-based sampling and likelihood maximization. (Fuller, 1987, pp. 103-106; Bound, Brown and Mathiowetz, 2001). Here this would require (a) $\tau_{k i}$ and $U_{k i}$ to be normally distributed over our census and (b) the error sum of squares over this census, $\delta=\sum_{p} U_{k i}^{2}$ in (3.4), to be known. Important advantages of true-value theory are that it requires no (dubious) distribution assumptions about true and error scores and it estimates $\delta$ in (3.4) by $d$ in (5.2). This allows measurement error to be circumvented by equation (5.1) which uses Särndal-Lundström estimation of census totals. Table 3 shows that this SL estimation simultaneously corrects an unrepresentative sub-sample for measurement error and unit non-response. Thus, true-value theory, with respondentspecific weight calibration and imputation, resolves four major issues in survey regression without any distribution assumptions; namely, errors in variables, equation error, item non-response and unit non-response.

\section{Acknowledgements}

This work is dedicated to Min-Te Chao, the JDS founding editor, who motivated me to investigate non-response in survey sampling. It was supported by the University of Florida's Warrington College of Business Administration and is an extension of work appearing in Bechtel (2010). The cross-national regressions were obtained by user-friendly access to round four of the European Social Survey. Norwegian Social Science Data Services (NSD) is the data archive and distributor of the ESS data. None of the ideas or analyses in the present paper may be attributed to the European Social Survey or the University of Florida.

\section{References}

Bechtel, G. G. (2010). True-value regression theory. Journal of Data Science 8, 521-539.

Bechtel, T. G. (2007). The pursuit of happiness. Survey Research Methods 1, 109-120.

Bellhouse, D. R. (1988). A brief history of random sampling methods. In Handbook of Statistics, Volume 6 (Sampling) (Edited by P. R. Krishnaiah and C. R. Rao), 1-14. North Holland, New York.

Binder, D. A. (1983). On the variances of asymptotically normal estimators from complex samples. International Statistical Review 51, 279-292.

Bound, J., Brown, C. and Mathiowetz, N. (2001). Measurement error in survey data. In Handbook of Econometrics, Volume 5 (Edited by J. J. Heckman and E. Leamer), 3705-3843. Elsevier, Amsterdam.

Chaudhuri, A. and Stenger, H. (2005). Survey Sampling: Theory and Methods, 2nd edition. Chapman and Hall / CRC Press, New York. 
Cronbach, L. J. (1951). Coefficient alpha and the internal structure of tests. Psychometrika 16, 297-334.

Fuller, W. A. (1987). Measurement Error Models. Wiley, New York.

Gulliksen, H. (1950). Theory of Mental Tests. Wiley, New York.

Jowell, R. and the Central Co-ordinating Team. (2008). European Social Survey 2008/2009: Technical Reports. Centre for Comparative Social Surveys, City University, London.

Lehmann, E. L. (1999). Elements of Large-Sample Theory. Springer, New York.

Lohr, S. L. (1999). Sampling: Design and Analysis. Duxbury Press, Pacific Grove, CA.

Lord, F. M. and Novick, M. R. (1968). Statistical Theories of Mental Test Scores. Addison-Wesley, Menlo Park, CA.

Nathan, G. (1988). Inference based on data from complex sample designs. In Handbook of Statistics, Volume 6 (Sampling) (Edited by P. R. Krishnaiah and C. R. Rao), 247-266. North Holland, Elsevier, Amsterdam.

Neyman, J. (1934). On two different aspects of the representative method: The method of stratified sampling and the method of purposive selection. Journal of the Royal Statistical Society 97, 558-625.

Nunnally, J. C. and Bernstein, I. H. (1994). Psychometric Theory, 3rd edition. McGrawHill, New York.

Särndal, C. E. and Lundström, S. (2005). Estimation in Surveys with Nonresponse. Wiley, New York.

Skinner, C. J., Holt, D. and Smith, T. M. F. (1989). Analysis of Complex Surveys. Wiley, New York.

StataCorp. (2001). Stata Statistical Software: Release 7.0. Stata Corporation.

Thompson, M. E. (1997). Theory of Sample Surveys. Chapman \& Hall, London.

Valliant, R., Dorfman, A. H. and Royall, R. M. (1999). Finite Population Sampling and Inference: A Prediction Approach. Wiley, New York.

Received June 22, 2010; accepted December 20, 2010.

Gordon G. Bechtel

University of Florida and Florida Research Institute P.O. Box 117155, Gainesville, Florida 32611-7155, USA

bechtel@ufl.edu 